\title{
Chemical Composition and Antibacterial Properties of Achillea micrantha
}

\author{
O. ASTAFYEVA, L. SUKHENKO, E. KURASHOV ${ }^{1,2}$, J. KRYLOVA ${ }^{2,3}$, M. EGOROV, Y. BATAEVA AND A. BAIMUKHAMBETOVA \\ Department of Biotechnology, Faculty of Biology, Astrakhan State University, pl. Shaumyana 1, 414000, Astrakhan, \\ ${ }^{1}$ Laboratory of Hydrobiology of Institute of Limnology, Russian Academy of Sciences, ul.Sevastianova, 9, St. Petersburg, \\ 196105, ${ }^{2}$ Department of Ecology and Technosphere Safety, ITMO University, pr.Kronverskyi, 49, St. Petersburg, 197101, \\ ${ }^{3}$ Laboratory of Environmental Toxicology, Berg State Research Institute on Lake and River Fisheries, nab. Makarova, 26, \\ 199053, St.Petersburg, Russia
}

\section{Astafyeva, et al.: Chemical Composition and Antibacterial Properties of Achillea micrantha}

\begin{abstract}
The composition of detectable small organic compounds in the ethanol extract of Achillea micrantha was defined by means of gas chromatography-mass spectrometric analysis. There were 71 low molecular weight organic compounds observed, two of which remained unidentified. The antibacterial activity of the extract was studied in respect to Escherichia coli, Staphylococcus aureus, Pseudomonas aeruginosa using the agar diffusion test and serial dilutions to define minimum inhibitory concentration. In order to compare the antibacterial activity of the herb and blossom truss extract of Achillea micrantha, the extracts of Achillea millefolium and Achillea leptophylla was used. In relation to the microorganisms tested, a significant inhibitory effect was observed with the aqueous alcoholic extract of Achillea micrantha at the minimum inhibitory concentration of $0.05 \mu \mathrm{g} / \mathrm{ml}$.
\end{abstract}

Key words: Achillea micrantha, low molecular weight organic compounds, gas chromatography-mass spectrometry, antibacterial activity

The genus Achillea of compositae (Asteraceae) contains about 150 species. It vegetates mostly on the territory of Europe and Western Asia as well as in Australia, New Zealand and North America ${ }^{[1]}$. Different scientists managed to extract some chemical substances from the blossom trussand herb ofyarrow, Achillea millefolium L., such as lactones, achillin, artillizin, grossmizin, michrantin, kaempferol 3-rhamnoside, campesterol ${ }^{[2,3]}$, sesquiterpene lactones, sinthenin and micrantin ${ }^{[4,5]}$, other various components of essential oils ${ }^{[6,7]}$ and flavonoids ${ }^{[8]}$.

The flavonoid content in the yarrow was considered to make antipyretic and haemostatic drugg ${ }^{[9]}$. In ethnomedicine, the herb and tips of flowering plants (anthodes) are used as a haemostatic agent in cases of internal haemorrhage, gastric disorders, external haemorrhage, inflammatory processes and metabolopathy ${ }^{[10-12]}$. There were reports of antioxidant activity of the extracts and essential oils of the plants sp. Achillea ${ }^{[13-15]}$, as well as estrogenic ${ }^{[16]}$, antiulcer $^{[17,18]}$, antitumoral ${ }^{[19]}$, antisecretory (inhibiting intestinal motility) ${ }^{[20]}$ immunomodulatory ${ }^{[21]}$, fibrinogenic ${ }^{[22]}$ antimicrobial ${ }^{[23-27]}$, antifungal ${ }^{[28]}$,

*Address for correspondence

E-mail: astra39@list.ru

May-June 2018

Indian Journal of Pharmaceutical Sciences and antiinflammatory ${ }^{[29,30]}$ activities. In traditional medicine, in contrast to A. micrantha Willd., the herb and blossom truss of A. millefolium, the chemical composition of which has been properly studied is mostly used. Nevertheless, literature reported that the antimicrobial effect of the extracts A. micrantha was much higher than that of A. millefolium ${ }^{[23]}$. The aim of this paper was to examine the chemical composition of the extracts of $A$. micrantha vegetative elements and blossom truss and their antibacterial activity in comparison with the extracts of other Achillea species.

\section{MATERIALS AND METHODS}

Gas chromatography/mass spectrometry (GC/MS) analysis:

The low-molecular weight organic compounds (LMWOC) of the extracts were defined by methods of

This is an open access article distributed under the terms of the Creative Commons Attribution-NonCommercial-ShareAlike 3.0 License, which allows others to remix, tweak, and build upon the work non-commercially, as long as the author is credited and the new creations are licensed under the identical terms

Accepted 15 March 2018

Revised 03 August 2017

Received 07 September 2016

Indian J Pharm Sci 2018;80(3):434-441 
GC/MS with the help of Shimadzu GC/MS (QP-5050A, Shimadzu, Japan) equipped with the software Class 5000 and chromato-mass spectrometer TRACE DSQ II (Thermo Electron Corporation) with quadrupole mass analyser. In the first case, the column of DBI, 30 $\mathrm{m} ; 0.53 \mathrm{~mm}$ ID и $1.5 \mu \mathrm{m}$ film (J\&W Scientific) was used. As a mobile phase the carrier gas helium was applied. Ionization mode- EI, ionization voltage $-70 \mathrm{ev}$. Temperature program: $5^{\circ}(1 \mathrm{~min})-150^{\circ}(1 \mathrm{~min})$ at $10^{\circ} \%$ $\min -250^{\circ}(5 \mathrm{~min})$ at $5^{\circ} / \mathrm{min}-270^{\circ}(2 \mathrm{~min})$ at $3.5^{\circ} / \mathrm{min}$. Detector temperature was set at $250^{\circ}$ and the injector temperature was set at $280^{\circ}$.

In the second one, before the analysis started the preparation was 200-fold attenuated and transformed into hexane. The constitution of the compounds in the solution obtained was detected by means of the chromatomass spectrometer with quadrupole mass analyser. The column Thermo TR-5 ms SQC $15 \mathrm{~m} \times 0.25 \mathrm{~mm}$ phase ID 0.25 micron was applied. As the carrier gas helium was used. The mass spectra were tested in mode of scanning on mass full-scale range (30$580 \mathrm{~m} / \mathrm{z})$ at a programmed temperature range $\left(35^{\circ}\right.$ $3 \mathrm{~min}, 2^{\circ} / \mathrm{min}$ to $60^{\circ}-3 \mathrm{~min}, 2^{\circ} / \mathrm{min}$ to $80^{\circ}-3 \mathrm{~min}, 4^{\circ} / \mathrm{min}$ to $120^{\circ}-3 \mathrm{~min}, 5^{\circ} / \mathrm{min}$ to $150^{\circ}-3 \mathrm{~min}, 15^{\circ} / \mathrm{min}$ to $240^{\circ}$ $10 \mathrm{~min}$ ) with the subsequent step-by-step operation of chromatograms. The agents detected were identified with the help of mass spectral libraries (NIST-2005 and Wiley). For more precise identification the Kavats's retention index, obtained due to the use of the standards of alkanes $\mathrm{C}_{7}-\mathrm{C}_{30}$, was applied. The quantitative analysis was made through the internal standards, decafluorobenzophenone and benzophenone.

\section{Plant material:}

The blossom trusses, leaves and footstalks of A. micrantha were picked in the spring-summer period (May-June) of 2010-11 at blossom-time on the territory of Volga Sands, Astrakhan region, Russia. Species identification was carried visually and microscopically by examining the morphology of blossoms in trusses, leaves and other characteristic features of the plants, and defined them with the determiner at the Botany Department, Astrakhan State University. While determining the antibacterial activity of $A$. micrantha extracts, the known medical plants of Achillea genus, A. millefolium, as well as A. leptophylla Bieb., growing in Astrakhan region were used for comparison.

The dried plant raw material (blossom trusses, leaves and footstalks) was extracted with $40 \%$ aqueous solution of ethanol at an indoor temperature during $7 \mathrm{~d}$ while being constantly stirred. Then the extract was filtered, with the spirit evaporated, pasteurized in the dry-air sterilizer at a temperature of $85^{\circ}$. The final product was used to examine the antibacterial activity of the extract obtained. In the course of our pasteurization of extracts, some of the organic molecules could be destroyed due to the high temperature, and they were ultimately not taken into account in the analysis. However, this was done in order to obtain an extract completely free of microorganisms for further experiments.

\section{Antibacterial assay:}

The antibacterial activity of the extracts was tested using the agar diffusion test and counting the colony-forming units (CFUs) of test organisms, Staphylococcus aureus Rosenbach RNCIM B-1899, Escherichia coli Migula CK RNCIM B-1911, obtained from the Russian National Collection of Industrial Microorganisms, FSUE, State Research Institute of Genetics (Moscow, Russia); Pseudomonas aeruginosa Migula 1315, Staphylococcus epidermidis Rosenbach 537, obtained from the regional infectious hospital. The minimum inhibitory concentration (MIC) was tested by means of count method of CFUs on solid medium. Gentamycin was used as the standard drug.

\section{RESULTS AND DISCUSSION}

In the study of compositional analysis of aqueous alcoholic extracts of $A$. micrantha vegetative elements (leaves and footstalks) through $\mathrm{GC} / \mathrm{MS}$, there were a large number of LMWOC related to different groups of chemical compounds (Tables 1 and 2). Here with, aldehydes, alcohols and hydrocarbons were found prevailing (Table 2). Out of 55 defined compounds, one remained unidentified (fig. 1). The concentration of two compounds (butane-1,3-diol and hexanal) was the highest. Their proportion was about $63 \%$ from the total LMWOC concentration in the solution. Hexanal participates in regulation of different plant reactions, among them the formation of plant defence mechanisms from external damage and plant-feeders ${ }^{[31-33]}$. This compound has antifungal and antimicrobial properties ${ }^{[34]}$. The substantial part of all LMWOCs were aromatic compounds and benzene derivatives (Table 1). The most abundant of them are given in fig. 2. All these compounds are bioactive.

It is interesting to have found some compounds, naphthalene derivatives (compounds 29, 30, 32, 33 , Table 1), the share of which is $2.75 \%$ of the 


\begin{tabular}{|c|c|c|c|c|c|c|}
\hline Peak & Compound & Formula & RT & IK & $\bar{C}$ & $\%$ \\
\hline 1 & hexan-2-one & $\mathrm{C}_{6} \mathrm{H}_{12} \mathrm{O}$ & 2.55 & 796 & 183.47 & 1.93 \\
\hline 2 & butane-1,3-diol & $\mathrm{C}_{4} \mathrm{H}_{10} \mathrm{O}_{2}$ & 2.72 & 803 & 2000.78 & 21.08 \\
\hline 3 & Hexanal & $\mathrm{C}_{6}^{4} \mathrm{H}_{12} \mathrm{O}$ & 2.97 & 812 & 3980.44 & 41.93 \\
\hline 4 & Ethylbenzene & $\mathrm{C}_{8} \mathrm{H}_{10}$ & 4.11 & 853 & 68.31 & 0.72 \\
\hline 5 & 1,4-dimethylbenzene & $\mathrm{C}_{8} \mathrm{H}_{10}$ & 4.34 & 861 & 279.39 & 2.94 \\
\hline 6 & 3-methyloctane & $\mathrm{C}_{9} \mathrm{H}_{20}$ & 4.51 & 867 & 27.67 & 0.29 \\
\hline 7 & Ethenylbenzene [styrene] & $\mathrm{C}_{8} \mathrm{H}_{8}$ & 5.03 & 886 & 86.04 & 0.91 \\
\hline 8 & $\begin{array}{l}\text { 1,2-dimethylbenzene } \\
\text { [o-xylene] }\end{array}$ & $\mathrm{C}_{8} \mathrm{H}_{10}$ & 5.08 & 888 & 89.39 & 0.94 \\
\hline 9 & 5-methoxypentan-2-one & $\mathrm{C}_{6} \mathrm{H}_{12} \mathrm{O}_{2}$ & 5.9 & 911 & 65.49 & 0.69 \\
\hline 10 & 1-(4-methylpentan-2-yloxy)propan-2-ol & $\mathrm{C}_{9} \mathrm{H}_{20} \mathrm{O}_{2}$ & 6.05 & 914 & 303.89 & 3.2 \\
\hline 11 & 4,7,7-trimethylbicyclo[4.1.0]hept-3-ene [4-carene] & $\mathrm{C}_{10} \mathrm{H}_{16}$ & 6.58 & 925 & 31.59 & 0.33 \\
\hline 12 & 1,2,3-trimethylbenzene & $\mathrm{C}_{9} \mathrm{H}_{12}$ & 9.38 & 986 & 144.93 & 1.53 \\
\hline 13 & (2S)-2-tert-butyl-5-methylidene-1,3-dioxolan-4-one & $\mathrm{C}_{8} \mathrm{H}_{12} \mathrm{O}_{3}$ & 10 & 999 & 186.55 & 1.97 \\
\hline 14 & 3,7,7-trimethylbicyclo[4.1.0]hept-3-ene [3-carene ] & $\mathrm{C}_{10} \mathrm{H}_{16}$ & 10.26 & 1004 & 37 & 0.39 \\
\hline 15 & $\begin{array}{l}\text { Unidentified compounds } \\
\mathrm{m} / \mathrm{z} 139\left[\mathrm{M}^{+}\right], 59(100)\end{array}$ & & 10.81 & 1013 & 70.48 & 0.74 \\
\hline 16 & 1-methyl-3-propan-2-ylbenzene [m-cymene] & $\mathrm{C}_{10} \mathrm{H}_{14}$ & 11.08 & 1017 & 70.39 & 0.74 \\
\hline 17 & 1-methyl-4-prop-1-en-2-ylcyclohexene [d-limonene] & $\mathrm{C}_{10} \mathrm{H}_{16}$ & 11.3 & 1021 & 25.81 & 0.27 \\
\hline 18 & 5-ethyl-2,2,3-trimethylheptane & $\mathrm{C}_{12} \mathrm{H}_{26}$ & 11.36 & 1022 & 50.5 & 0.53 \\
\hline 19 & 1-methyl-4-propan-2-ylcyclohexa-1,4-diene [ $\gamma$-Terpinene] & $\mathrm{C}_{10} \mathrm{H}_{16}$ & 13.13 & 1052 & 25.88 & 0.27 \\
\hline 20 & 2-methyldecane & $\mathrm{C}_{11} \mathrm{H}_{24}$ & 13.63 & 1060 & 51.34 & 0.54 \\
\hline 21 & 3,7-dimethyldecane & $\mathrm{C}_{12} \mathrm{H}_{26}$ & 14.21 & 1070 & 37.98 & 0.4 \\
\hline 22 & (6S,7aS)-6-Ethyl-hexahydro-pyrrolizin-3-one & $\mathrm{C}_{9} \mathrm{H}_{15} \mathrm{NO}$ & 14.43 & 1073 & 41.22 & 0.43 \\
\hline 23 & 2,3,4-trimethyldecane & $\mathrm{C}_{13} \mathrm{H}_{28}$ & 15.08 & 1084 & 29.85 & 0.31 \\
\hline 24 & 4-tert-butyl-1,3-thiazole & $\mathrm{C}_{7} \mathrm{H}_{11} \mathrm{NS}$ & 15.76 & 1096 & 22.06 & 0.23 \\
\hline 25 & 3,3-diethyl-4,5-dimethylhex-4-en-2-one & $\mathrm{C}_{12} \mathrm{H}_{22} \mathrm{O}$ & 16.91 & 1111 & 42.16 & 0.44 \\
\hline 26 & 1,1,3,3,5-pentamethylcyclohexane & $\mathrm{C}_{11} \mathrm{H}_{22}$ & 17.81 & 1123 & 25.9 & 0.27 \\
\hline 27 & 1,5-diethyl-2,3-dimethylcyclohexane & $\mathrm{C}_{12} \mathrm{H}_{24}$ & 18.46 & 1131 & 54.05 & 0.57 \\
\hline 28 & 1-methyl-2-pentylcyclohexane & $\mathrm{C}_{12} \mathrm{H}_{24}$ & 19.57 & 1145 & 40.84 & 0.43 \\
\hline 29 & 2,3-dimethyl-1,2,3,4,4a,5,6,7,8,8a-decahydronaphthalene & $\mathrm{C}_{12}^{12} \mathrm{H}_{22}$ & 22.17 & 1178 & 29.25 & 0.31 \\
\hline 30 & 1,6-dimethyl-1,2,3,4,4a,5,6,7,8,8a-decahydronaphthalene & $\mathrm{C}_{12} \mathrm{H}_{22}$ & 22.77 & 1186 & 15.36 & 0.16 \\
\hline 31 & dodecane & $\mathrm{C}_{12} \mathrm{H}_{26}$ & 23.9 & 1200 & 106.91 & 1.13 \\
\hline 32 & 2,6-dimethyl-1,2,3,4,4a,5,6,7,8,8a-decahydronaphthalene & $\mathrm{C}_{12}^{12} \mathrm{H}_{22}$ & 24.17 & 1204 & 119.9 & 1.26 \\
\hline 33 & 1,5-dimethyl-1,2,3,4,4a,5,6,7,8,8a-decahydronaphthalene & $\mathrm{C}_{12} \mathrm{H}_{22}$ & 24.58 & 1209 & 97.07 & 1.02 \\
\hline 34 & 4,10-dimethylspiro[4.5]decane & $\mathrm{C}_{12} \mathrm{H}_{22}$ & 25.72 & 1224 & 24.26 & 0.26 \\
\hline 35 & tetradecane & $\mathrm{C}_{14} \mathrm{H}_{30}$ & 37.59 & 1400 & 60.17 & 0.63 \\
\hline 36 & dodecanoyl chloride & $\mathrm{C}_{12} \mathrm{H}_{23} \mathrm{ClO}$ & 39.35 & 1443 & 12.64 & 0.13 \\
\hline 37 & 2,6-ditert-butyl-4-methylphenol & $\mathrm{C}_{15} \mathrm{H}_{24} \mathrm{O}$ & 41.8 & 1504 & 14.25 & 0.15 \\
\hline 38 & hexadecane & $\mathrm{C}_{16} \mathrm{H}_{34}$ & 45.93 & 1600 & 19.07 & 0.2 \\
\hline 39 & 4-pyrrolidin-1-ylbenzene-1,3-diol & $\mathrm{C}_{10} \mathrm{H}_{13} \mathrm{NO}_{2}$ & 47.06 & 1632 & 9.25 & 0.1 \\
\hline 40 & undecylcyclopentane & $\mathrm{C}_{16} \mathrm{H}_{32}$ & 47.68 & 1650 & 7.16 & 0.08 \\
\hline 41 & octadecane & $\mathrm{C}_{18} \mathrm{H}_{38}$ & 52.25 & 1800 & 14.7 & 0.15 \\
\hline 42 & $\begin{array}{l}\text { bis(2-methylpropyl) benzene-1,2-dicarboxylate } \\
\text { [diisobutyl phthalate] }\end{array}$ & $\mathrm{C}_{16} \mathrm{H}_{22} \mathrm{O}_{4}$ & 54.31 & 1871 & 24.67 & 0.26 \\
\hline 43 & (z)-hexadec-11-enoic acid & $\mathrm{C}_{16} \mathrm{H}_{30} \mathrm{O}_{2}$ & 55.72 & 1945 & 13.23 & 0.14 \\
\hline 44 & $\begin{array}{l}\text { dibutyl benzene-1,2-dicarboxylate } \\
\text { [dibutyl phthalate] }\end{array}$ & $\mathrm{C}_{16} \mathrm{H}_{22} \mathrm{O}_{4}$ & 55.92 & 1960 & 71.54 & 0.75 \\
\hline 45 & hexadecanoic acid & $\mathrm{C}_{16} \mathrm{H}_{32} \mathrm{O}_{2}$ & 56.02 & 1968 & 76.83 & 0.81 \\
\hline 46 & ethyl hexadecanoate & $\mathrm{C}_{18} \mathrm{H}_{36} \mathrm{O}_{2}$ & 56.36 & 1993 & 14.62 & 0.15 \\
\hline 47 & eicosane & $\mathrm{C}_{20} \mathrm{H}_{42}$ & 56.45 & 2000 & 24.02 & 0.25 \\
\hline
\end{tabular}




\begin{tabular}{|c|c|c|c|c|c|}
\hline 48 & $\begin{array}{c}\text {-[(1S,4aS, 8aS)-5,5,8a-trimethyl-2-methylidene-3,4,4a,6,7,8-hexahydro- } \\
\text { 1H-naphthalen-1-yl]-3-methylpent-1-en-3-ol; } \\
\text { [manool] }\end{array}$ & $\mathrm{C}_{20} \mathrm{H}_{34} \mathrm{O}$ & 56.872044 & 15.26 & 0.16 \\
\hline 49 & icos-1-ene & $\mathrm{C}_{20} \mathrm{H}_{40}$ & 57.042062 & 24.72 & 0.26 \\
\hline 50 & methyl 10 -octadecenoate & $\mathrm{C}_{19} \mathrm{H}_{36} \mathrm{O}_{2}$ & 57.692137 & 80.26 & 0.85 \\
\hline 51 & docosane & $\mathrm{C}_{22} \mathrm{H}_{46}$ & 58.192200 & 47.85 & 0.5 \\
\hline 52 & docos-1-ene & $\mathrm{C}_{22} \mathrm{H}_{44}$ & 58.632266 & 72.02 & 0.76 \\
\hline 53 & bis(2-ethylhexyl) hexanedioate & $\mathrm{C}_{22} \mathrm{H}_{42} \mathrm{O}_{4}$ & 59.432392 & 41.6 & 0.44 \\
\hline 54 & $\begin{array}{l}\text { bis(2-ethylhexyl) benzene-1,2-dicarboxylate } \\
\text { [diethylhexyl phthalate] }\end{array}$ & $\mathrm{C}_{24} \mathrm{H}_{38} \mathrm{O}_{4}$ & 60.452538 & 72.06 & 0.76 \\
\hline 55 & $\begin{array}{c}(6 \mathrm{E}, 10 \mathrm{E}, 14 \mathrm{E}, 18 \mathrm{E})-2,6,10,15,19,23 \text {-hexamethyltetracosa-2,6,10,14,18,22- } \\
\text { hexaene } \\
\text { [squalene] }\end{array}$ & $\mathrm{C}_{30} \mathrm{H}_{50}$ & $63.65 \quad 2814$ & 311.29 & 3.28 \\
\hline
\end{tabular}

RT: Retention time, min, IK - Kovats retention index; C - concentration of the compounds in the extract, $\mathrm{mg} \mathrm{l}^{-1}$

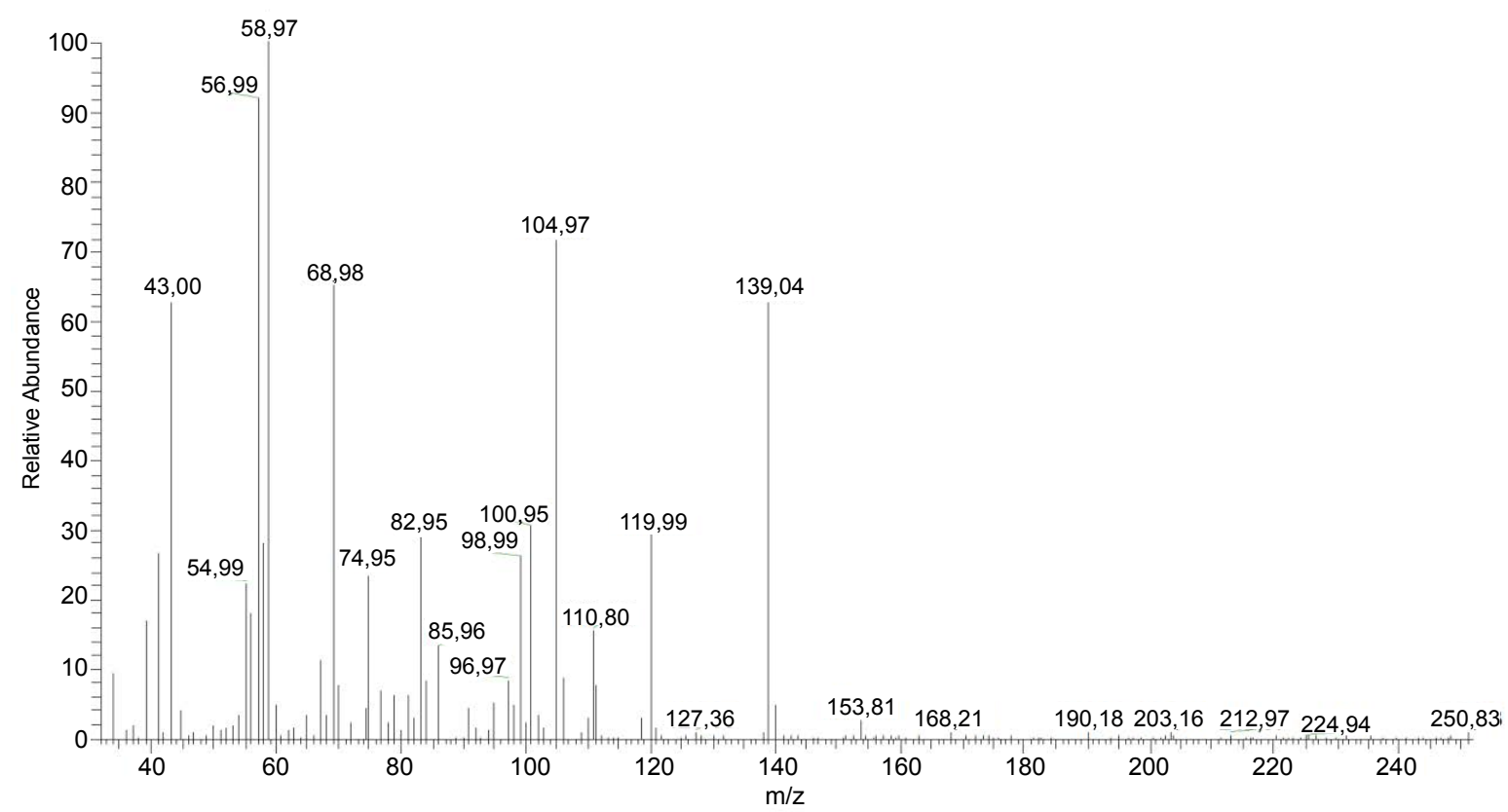

Fig. 1: Mass spectrometry of the unidentified compound RT=10.81 $\mathrm{min}$

TABLE 2: PERCENT RELATIVE CONTENT OF THE MAIN GROUPS OF SUBSTANCES IN THE AQUEOUS ETHANOLIC EXTRACT OF VEGETATIVE PARTS OF A. MICRANTHA

\begin{tabular}{lc}
\hline Group of substances & Relative content, \% \\
\hline Alcohols & 21.24 \\
Hydrocarbons & 14.45 \\
Esters & 3.21 \\
Ketones & 2.37 \\
Aldehydes & 41.93 \\
Diverse functional groups & 6.29 \\
Aromatic hydrocarbons & 7.78 \\
Nitrogen and sulphur containing & 0.76 \\
compounds & 0.13 \\
Chlorine-containing compounds & 0.95 \\
Carboxylic acids & 0.15 \\
Phenols & 0.74 \\
Unidentified compounds &
\end{tabular}

total concentration of LMWOC. Among LMWOCs of vegetative parts extract of $A$. micrantha, three compounds related to phthalates were identified (diisobutyl phthalate, dibutyl phthalate, diethylhexyl phthalate); herewith their total share was quite large $(1.77 \%)$. The given group of substances is often considered as pollutants. However, the recent findings have shown that actinomycetes, fungus and plants (terraneous and aquatic ones) are able to synthesize phthalates, which participate in allelopathic interacting and perform protective functions ${ }^{[35-39]}$.

In ethanol extract of $A$. micrantha truss, 19 compounds were identified (Table 3). Out of the 19 compounds, identified in the truss extract of milfoil Parviflorus, 17 related to terpenes and their derivatives. This fact can be indicative of substantial antibacterial activity of this 
A.<smiles>CCc1ccccc1</smiles><smiles>Cc1ccccc1C</smiles>

D.

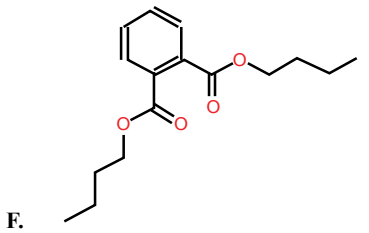

B.

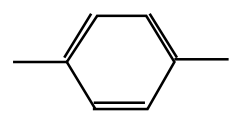

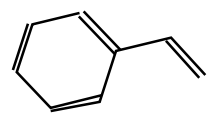<smiles>Cc1cccc(C)c1C</smiles>

E.

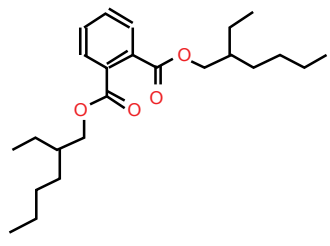

Fig. 2: The most abundant aromatic compounds and benzene derivatives in the aqueous ethanol extract of vegetative parts of $A$. micrantha

A. Ethylbenzene, B. 1,4-dimethylbenzene, C. ethenylbenzene, D. 1,2-dimethylbenzene, E. 1,2,3-trimethylbenzene, F. dibutyl phthalate, G. diethylhexyl phthalate

TABLE 3: PERCENT RELATIVE CONTENT OF LMWOC IN THE AQUEOUS ETHANOLIC (40 \% V/V) EXTRACT OF INFLORESCENCES OF A. MICRANTHA

\begin{tabular}{|c|c|c|c|c|}
\hline No. & Compound & Formula & IK & $\begin{array}{c}\text { Relative } \\
\text { content, \% }\end{array}$ \\
\hline 1 & 4,6,6-trimethylbicyclo[3.1.1]hept-3-ene [a-pinene] & $\mathrm{C}_{10} \mathrm{H}_{16}$ & 939 & 2.46 \\
\hline 2 & 3,3-dimethyl-2-methylidenebicyclo[2.2.1]heptane [camphene] & & 945 & 0.5 \\
\hline 3 & 4-methylidene-1-propan-2-ylbicyclo[3.1.0]hexane [sabinene] & & 974 & 0.1 \\
\hline 4 & 6,6-dimethyl-4-methylidenebicyclo[3.1.1] heptane [B-pinene] & & 982 & 2.1 \\
\hline 5 & 1-methyl-4-prop-1-en-2-ylcyclohexene [limonene] & $\mathrm{C}_{10} \mathrm{H}_{16}$ & 1029 & 1.3 \\
\hline 6 & 2,2,4-trimethyl-3-oxabicyclo[2.2.2]octane [1,8-cineol] & $\mathrm{C}_{10} \mathrm{H}_{18} \mathrm{O}$ & 1043 & 8.47 \\
\hline 7 & 1-methyl-4-propan-2-ylcyclohexa-1,4-diene [ $\gamma$-terpinene] & & 1079 & 4.9 \\
\hline 8 & 3,7-dimethylocta-1,6-dien-3-ol [linalool] & $\mathrm{C}_{10} \mathrm{H}_{18} \mathrm{O}$ & 1097 & 0.56 \\
\hline 9 & Unidentified & & 1132 & 2.93 \\
\hline 10 & 4,7,7-trimethylbicyclo[2.2.1] heptan-3-one [camphor] & $\mathrm{C}_{10} \mathrm{H}_{16} \mathrm{O}$ & 1156 & 10.62 \\
\hline 11 & 4,7,7-trimethylbicyclo[2.2.1]heptan-3-ol [borneol] & $\mathrm{C}_{10} \mathrm{H}_{18} \mathrm{O}$ & 1169 & 1.37 \\
\hline 12 & 2-(4-methylcyclohex-3-en-1-yl)propan-2-ol [terpineol] & $\mathrm{C}_{10} \mathrm{H}_{18} \mathrm{O}$ & 1174 & 1.53 \\
\hline 13 & (6S)-3-methyl-6-propan-2-ylcyclohex-2-en-1-one [piperitone] & $\mathrm{C}_{10} \mathrm{H}_{16} \mathrm{O}$ & 1224 & 34.15 \\
\hline 14 & (4,7,7-trimethyl-3-bicyclo[2.2.1]heptanyl) acetate [bornyl acetate] & $\mathrm{C}_{12} \mathrm{H}_{20} \mathrm{O}_{2}$ & 1237 & 1.32 \\
\hline 15 & 2-methyl-5-prop-1-en-2-ylcyclohex-2-en-1-one [carvone] & $\mathrm{C}_{10} \mathrm{H}_{14} \mathrm{O}$ & 1242 & 24.93 \\
\hline 16 & dibutyl benzene-1,2-dicarboxylate [dibutyl phthalate] & $\mathrm{C}_{16} \mathrm{H}_{22} \mathrm{O}_{4}$ & 1960 & 1.37 \\
\hline 17 & Octadecanoic acid & $\mathrm{C}_{18}^{10} \mathrm{H}_{36} \mathrm{O}_{2}$ & 2100 & 0.45 \\
\hline 18 & $\begin{array}{c}\text { (3S, } 8 \mathrm{~S}, 9 \mathrm{~S}, 10 \mathrm{R}, 13 \mathrm{R}, 14 \mathrm{~S}, 17 \mathrm{R})-17-[(2 \mathrm{R}, 5 \mathrm{R}) \text {-5-ethyl-6-methylheptan-2-yl]-10,13- } \\
\text { dimethyl-2,3,4,7,8,9,11,12,14,15,16,17-dodecahydro-1H-cyclopenta[a] } \\
\text { phenanthren-3-ol [B-sitosterol] }\end{array}$ & $\mathrm{C}_{29} \mathrm{H}_{50} \mathrm{O}$ & 3200 & 0.718 \\
\hline 19 & $\begin{array}{c}(3 \mathrm{~S}, 8 \mathrm{~S}, 9 \mathrm{~S}, 10 \mathrm{R}, 13 \mathrm{R}, 14 \mathrm{~S}, 17 \mathrm{R}) \text {-17-[(E,2R,5S)-5-ethyl-6-methylhept-3-en-2-yl]- } \\
\text { 10,13-dimethyl-2,3,4,7,8,9,11,12,14,15,16,17-dodecahydro-1H-cyclopenta[a] } \\
\text { phenanthren-3-ol [stigmasterol] }\end{array}$ & $\mathrm{C}_{29} \mathrm{H}_{48} \mathrm{O}$ & 3230 & 0.24 \\
\hline
\end{tabular}

IK: Kovats retention index

extract, as well as of vegetative parts extract where the proportion of terpenoids is also big, as the high antibacterial activity is intrinsic for terpenes and their derivatives $^{[40-42]}$.

The given chemical compounds, the majority of which refer to the group of biologically active terpenes and their derivatives were extracted with $40 \%$ ethanol from the yarrow blossom trusses, were also the ones most commonly found in essential oils. The offered preparation method of extracting these compounds with the help of ethanol made it possible to obtain a complex of organic substances, which were the constituents of some essential oils with antimicrobial properties.

The complex of the detected compounds in the extracts A. micrantha had a notable inhibitory effect on the test strains studied. As evidenced from the Table 4, the 
TABLE 4: THE COMPARATIVE ANTIMICROBIALACTIVITY OF THE PLANT EXTRACTS BY AGAR DIFFUSION TEST

\begin{tabular}{lcccc}
\hline \multirow{2}{*}{ Samples } & \multicolumn{4}{c}{ Diameter of zone of inhibition M $\pm \mathrm{m} . \mathrm{mm}$} \\
\cline { 2 - 5 } & S. aureus & P. aeruginosa & E. coli & S. epidermidis \\
\hline Gentamycin & $42.0 \pm 1.4$ & $26.0 \pm 0.8$ & $16.0 \pm 0.5$ & $33.2+0.3$ \\
A. millefolium Bl. truss & $15.2 \pm 1.8$ & $12.4 \pm 1.2$ & $10.0 \pm 0.4$ & $22.8 \pm 0.8$ \\
A. millefolium leaf & $14.1 \pm 1.5$ & $11.8 \pm 0.9$ & $7.0 \pm 1.7$ & - \\
A. millefolium herb & $13.4 \pm 2.3$ & $13.0 \pm 1.3$ & - & - \\
A. micranta Bl. truss & $39.0 \pm 0.4$ & $21.0 \pm 0.8$ & $14.0 \pm 1.5$ & $25.2+1.5$ \\
A. micranta leaf & $17.3 \pm 1.2$ & $16.6 \pm 1.4$ & $10.0 \pm 2.4$ & - \\
A. micranta herb & $20.5 \pm 1.2$ & $19.5 \pm 1.0$ & $9.7 \pm 1.3$ & - \\
\hline
\end{tabular}

TABLE 5: MINIMUM INHIBITORY CONCENTRATION OF THE PLANT EXTRACTS IN TERMS OF STAPHYLOCOCCUS AUREUS

\begin{tabular}{lcc}
\hline Concentration of & \multicolumn{2}{c}{ CFUs. M $\pm \mathrm{m}_{\mathrm{m}}$} \\
\cline { 2 - 3 } extracts active & A. millefolium & A. micrantha \\
\hline Control without & $158.4 \pm 1.3$ & $213.2 \pm 2.5$ \\
extract & $50 \pm 0.6$ & $26.8 \pm 0.9$ \\
$0.25 \mu \mathrm{g} / \mathrm{ml}$ & $15.8 \pm 0.7$ & $17 \pm 0.9$ \\
$0.5 \mu \mathrm{g} / \mathrm{ml}$ & $3.6 \pm 0.2$ & $8.6 \pm 0.5$ \\
$2.5 \mu \mathrm{g} / \mathrm{ml}$ & $1.7 \pm 0.2$ & $4.7 \pm 1.03$ \\
$5.0 \mu \mathrm{g} / \mathrm{ml}$ &
\end{tabular}

CFU counting method $-1.0 \times 10^{8}$

TABLE 6: THE COMPARATIVE ANTIMICROBIAL ACTIVITY (MIC) OF PLANT EXTRACTS IN RESPECT OF STAPHYLOCOCCUS AUREUS

\begin{tabular}{|c|c|c|c|}
\hline \multirow{2}{*}{ Samples } & \multicolumn{3}{|c|}{$\left(M_{ \pm} m_{m}\right) \cdot m m$} \\
\hline & $5.0 \mu \mathrm{g} / \mathrm{ml}$ & $0.5 \mu \mathrm{g} / \mathrm{ml}$ & $0.05 \mu \mathrm{g} / \mathrm{ml}$ \\
\hline A. micrantha & $39.0 \pm 0.4$ & $20.0 \pm 0.5$ & $25.2 \pm 0.8$ \\
\hline A. millefolium & $16.5 \pm 0.2$ & $10.1 \pm 0.1$ & 0 \\
\hline A. leptophylla & $20.5 \pm 1.2$ & 0 & 0 \\
\hline Gentamycin & $42.0 \pm 1.4$ & $22.5 \pm 0.1$ & $10.0 \pm 0.1$ \\
\hline
\end{tabular}

most inhibitory effect on $S$. aureus was found in the A. micrantha blossom truss extract similar to the effect produced by gentamycin. The extracts of other parts of A. micrantha featured stronger antibacterial effect than the ones of $A$. millefolium. In this study the extracts of $A$. micrantha have demonstrated more antibacterial activity towards the strain $S$. aureus.

Besides, the study of effect of different concentrations (MIC) of the extracts A. micrantha and A. millefolium on the strain $S$. aureus (CFU counting method) showed more intense inhibiting effect of extract dilution A. micrantha (Table 5). The most active concentrations in MIC proved to be 5.0, 2.5, $0.25 \mu \mathrm{g} / \mathrm{ml}$. It should be noted, that the antibacterial activity of the concentration of $A$. micrantha $0.025 \mu \mathrm{g} / \mathrm{ml}$ was 1.8 as high as that of the extract A. millefolium (Table 5). The study of different extract concentrations A. millefolium, A. micrantha and A. leptophylla by means of agar diffusion test also revealed more intense inhibiting effect of the extract $A$. micrantha in respect of $S$. aureus (MIC value= 5.0, $0.05 \mu \mathrm{g} / \mathrm{ml}$; Table 6). As it was detected, the extracts of $A$. micrantha in all the concentrations studied 5.0, 0.5 and $0.05 \mu \mathrm{g} /$ $\mathrm{ml}$ showed more antibacterial action than those of A. millefoluim and A. leptophylla. The inhibitory activity of the extract is similar (compared to) to that of gentamycin, and the antibacterial action of the extract with 0.05 concentrations is 2.5 times higher than that of gentamycin (Table 6).

As per the results of the study, the detected antimicrobial action of the extract A. micrantha, similar due to its activity to chemical specific antibiotic, is determined by the content of terpenic and phenolic compounds as its major constituents, and other detected in the course of study compounds.

\section{Conflict of interests}

None declared.

Financial support and sponsorship:

Nil.

\section{REFERENCES}

1. Khani A, Asghari J. Insecticide activity of essential oils of Mentha longifolia, Pulicaria gnaphalodes and Achillea wilhelmsii against two stored product pests, the flour beetle, Tribolium castaneum and the cowpea weevil, Callosobruchus maculates. J Insect Sci 2012;12:73.

2. Hatam NAR, Yousif NJ, Porzel A, Seifert K. Sesquiterpene lactones from Achillea micrantha. Phytochemistry 1992;21(6):2160-62.

3. Souza TM, Rangel VLBI, Pietro Rr RCL, Santos LE, Moreira RRD. Phytochemical screening of Achillea millefolium harvested at Araraquara-Sp. Rev Bras Plantas Med 2006;8:151-54.

4. Rustaiyan A, Sharif Z, Tajarodi A, Sadjadi AS. Sesquiterpene lactones from Achillea micrantha. Phytochemistry 1987;26(10):2856-57.

5. Mahmoud AA, Al-Shihry SS, Hegazy MEF. A new epimeric 
sesquiterpene lactone from Achillea liguistica. Rec Nat Prod 2012;6(1):21-7.

6. Toncer O, Basbag S, Karaman S, Diraz E, Basbag M. Chemical composition of essential oils of some Achillea species growing wild in Turkey. Int J Agric Biol 2010;12:527-30.

7. Asimova SS, Glushenkova AI, Vinogradova VI. Lipids, lipophilic components and essential oils from plant sources. Berlin: Springer; 2012.

8. Valant-Vetschera KM. On the identity of five species of Achillea sect. Millefolium subsect Filipendulinae(Compositae, Anthemideae). Willdenowia 1999;29:141-46.

9. Benetis R, Radusiene J, Janulis V. Variability of phenolic compounds in flowers of Achillea millefolium wild populations in Lithuania. Medicina (Kaunas) 2008;44(10):775-81.

10. Priya KS, Gnanamani A, Radhakrishnan N, Babu M. Healing potential of Datura alba on burn wounds in albino rats. J Ethnopharmacol 2002;83:193-99.

11. Houghton PJ, Hylands PJ, Mensah AY, Hensel A, Deters AM. In vitro tests and ethnopharmacological investigations: wound healing as an example. J Ethnopharmacol 2005;100:100-07.

12. Saeidina S, Gohari AR, Mokhber-Derfuli N, Kiuchi F. A review on phytochemistry and medicinal properties of the genus Achillea. Daru 2011;19(3):173-86.

13. Konyalioglu S, Karamenderes CF. The protective effects of Achillea L. species native in Turkey against H2O2-induced oxidative damage in human erythrocytes and leucocytes. J Ethnopharmacol 2005;102:221-27.

14. Turkoglu I, Turkoglu S, Celik S, Kahyaoglu N. Antioxidant and antimicrobial activities of Turkish endemic Achillea species. African J Microbiol Res 2010;4(19):2034-42.

15. Vitalini S, Beretta G, Iriti M, Orsenigo S, Basilico N, Dall'Acqua S, et al. Phenolic compounds from Achillea millefolium L. and their bioactivity. Acta Biochim Pol 2011;58(2):203-9.

16. Innocentia G, Vegetob E, Dall-Acqua S, Cianab P, Giorgettia $\mathrm{M}$, Agradib E, et al. In vitro estrogenic activity of Achillea millefolium L. Phytomedicine 2007;14:147-52.

17. Niazmand S, Khoshnood E. The effects of Achillea wilhelmsii extract on rat's gastric motility at basal and vagal stimulated conditions. Iran J Basic Med Sci 2010;14(2):151-57.

18. Potrich FB, Allemand A, da Silva LM, dos Santos AC, Baggio $\mathrm{CH}$, Freitas $\mathrm{CS}$, et al. Antiulcerogenic activity of hydroalcoholic extract of Achillea millefolium L. involvement of the antioxidant system. J Ethnopharmacol 2010;130:85-92.

19. Lopes FCM, Benzatti FP, Junir CMJ, Moreira RRD, Carlos IZ. Effect of the essential oil of Achillea millefolium L. in the production of hydrogen peroxide and tumor necrosis factor- $\alpha$ in murine macrophages. Rev Bras Cienc Farm 2005;31(3):401-05.

20. Babaei M, Abarghoei ME, Akhavan MM, Ansari R, Vafaei AA, Taherian AA, et al. Antimotility effect of hydroalcolic extract of yarrow (Achillea millefolium) on the guinea-pig ileum. Pak J Biol Sci 2010;10(20):3673-77.

21. Sharififar F, Pournourmohammadi S, Arabnejad M. Immunomodulatiry activity of aqueous extract of Achillea wilhelmsii C. Kosh. in mice. Indian J Exp Biol 2009;47:668-71.

22. Hemmati AA, Arzi A, Adinehvand A, Mostofi NE, Mozaffari AR, Jalali A. Yarrow (Achillea millefolium L.) extract impairs the fibrogenic effect of bleomycin in rat lung. J Med Plants Res 2011;5(10):1843-49.

23. Sukhenko LT. Biologically active substances of some plants and the mechanisms of their antimicrobial activity. Nat Sci 2010;3:166-76.

24. Magiatis P, Skaltsouis A-L, Haroutounian SA. Chemical composition and in vitro antimicrobial activity of the essential oils of three Greek Achillea species. Z Naturforsch C 2002;57:287-90.

25. Kharma A, Hassawi D. The Antimicrobial activity and genetic relationship of Achillea species. Biotechnology 2006;5(4):501-07.

26. Baser KHC, Demirci B, Demirci F, Kocak S, Akinci C, Malyer $\mathrm{H}$, et al. Composition and antimicrobial activity of the essential oil of Achillea multifidi. Planta Med 2008;68:941-3.

27. Karaalp C, Yurtman AN, Yavasoglu NUK. Evaluation of antimicrobial properties of Achillea L. flower head extracts. Pharm Biol 2009;47(1):86-91.

28. Kordali S, Gakir A, Arkin TA, Mete E, Arkin A, Aydin T, et al. Antifungal and herbicidal properties of essential oils and n-hexane extracts of Achillea gypsicola Hub-Mor and Achillea biebersteinii Afan. (Asteraceae). Ind Crops Prod 2000;29:562-70.

29. Salvagnini LE, Migliato KF, Isaac VLB, Correa MA, Salgado NRN, Pietro RCLR. Evaluation of efficacy of preservatives associated with Achillea millefolium L. extract against Bacillus subtilis. Braz J Microbiol 2006;37:75-7.

30. Lakshimi T, Geetha RV, Roy A, Kumar SA. Yarrow (Achillea millefolium Linn.) a herbal medicanl plant with broad therapeutic use - a review. Int J Pharm Sci Rev Res 2011;9(2):136-41.

31. Fall R, Karl T, Hansel A, Jordan A, Lindinger W. Volatile organic compounds emitted after leaf wounding: On-line analysis by proton-transfer-reaction mass spectrometry. J Geophys Res 1999;104(13):15963-74.

32. Arimura G, Matsui K, Takabayashi J. Chemical and molecular ecology of herbivore-induced plant volatiles: proximate factors and their ultimate functions. Plant Cell Physiol 2009;50(5):911-23.

33. Jüttner F, Messina P, Patalano C, Zupo V. Odour compounds of the diatom Cocconeis scutellum: effects on benthic herbivores living on Posidonia oceanica. Mar Ecol Prog Ser 2010;400:63-73.

34. Lanciotti R, Belletti N, Patrignani F, Gianotti A, Gardini F, Guerzoni ME. Application of hexanal, (E)-2-hexenal, and hexyl acetate to improve the safety of fresh-sliced apples. J Agric Food Chem 2003;51:2958-63.

35. Xuan TD, Chung M, Khanh TD, Tawata S. Identification of Phytotoxic Substances from Early Growth of Barnyard Grass (Echinochloa crusgalli) Root Exudates. J Chem Ecol 2006;32:895-906.

36. Roy RN, Laskar S, Sen SK. Dibutyl phthalate, the bioactive compound produced by Streptomyces albidoflavus 321.2. Microbiol Res 2006;161(2):121-26.

37. Qiming X, Haidong C, Huixian Z, Daqiang Y. Chemical composition of essential oils of two submerged macrophytes, Ceratophyllum demersum L. and Vallisneria spiralis L. Flavour Fragr J 2006;21:524-26.

38. Qiming X, Haidong C, Huixian Z, Daqiang Y. Allelopathic activity of volatile substance from submerged macrophytes on Microcystin aeruginosa. Acta Ecologica Sinica 2006;26(11):3549-54.

39. Kurashov EA, Krylova JV, Mitrukova GG, Chernova AM. 
Low-molecular-weight metabolites of aquatic macrophytes growing on the territory of Russia and their role in hydroecosystems. Contemp Probl Ecol 2014;7(4):433-48.

40. Solís C, Becerra J, Flores C, Robledo J, Silva M. Antibacterial and antifungal terpenes from Pilgerodendron uviferum (D. Don) Florin. J Chil Chem Soc 2004;49(2):157-61.
41. Togashi N, Inoue Y, Hamashima H, Takano A. Effects of two terpene alcohols on the antibacterial activity and the mode of action of farnesol against Staphylococcus aureus. Molecules 2008;13:3069-76.

42. Gupta N, Saxena G, Kalra SS. Antimicrobial activity pattern of certain terpenoids. Int J Pharm Biol Sci 2011;2(1):87-91. 